\title{
Repeated metal ion measurements and long-term outcome of Durom/MMC total hip arthroplasty
}

\author{
Sakari PIETILÄINEN ${ }^{1,2}$, Erno SMEDBERG ${ }^{2}$, Inari LAAKSONEN ${ }^{1,2}$, Mikko S VENÄLÄINEN ${ }^{3,4}$, \\ Petteri LANKINEN ${ }^{1,2,5}$, and Keijo T MÄKELÄ 1,2
}

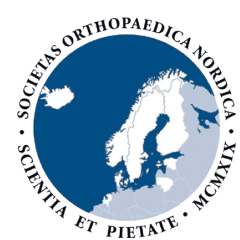

Acta Orthopaedica

\footnotetext{
${ }^{1}$ Department of Orthopaedics and Traumatology, Turku University Hospital; ${ }^{2}$ University of Turku; ${ }^{3}$ Turku Bioscience Centre, University of Turku and Åbo Akademi University; ${ }^{4}$ Division of Medical Imaging, Department of Medical Physics, Turku University Hospital, Turku; ${ }^{5}$ Department of Administration, Satakunta Central Hospital, Pori, Finland

Correspondence: sakari.pietilainen@gmail.com

Submitted 2021-06-21. Accepted 2021-12-19.
}

Background and purpose - Data regarding long-term behavior of metal ion levels in metal-on-metal total hip arthroplasty (MoM THA) patients is scarce. Therefore, we assessed whether there is any change in whole blood (WB) chromium $(\mathrm{Cr})$, and cobalt $(\mathrm{Co})$ ion measurements in Durom and MMC MoM THA patients over time. The secondary aim was to report the clinical outcomes using these devices in a single district.

Patients and methods - Durom and MMC cups were used in 249 MoM THAs from 2005 to 2011 in our district. Median follow-up time was 12 years for Durom THA (interquartile range $[\mathrm{IQR}]=3$ ) and 9 years for MMC THA (IQR = 1). A random coefficient model was used to compare individual differences in repeated WB Cr and Co ion measurements. The Kaplan-Meier estimator was used to analyze implant survival with any reason for revision as the endpoint.

Results - Geometric means of $\mathrm{Cr}$ in Durom THA and MMC THA patients decreased from $2.2 \mathrm{ppb}$ (geometric standard deviation $[\mathrm{SD}]=1.9$ ) to $1.5 \mathrm{ppb}$ (geometric $\mathrm{SD}=2.5, \mathrm{p}$ $<0.001$ ) and from $1.8 \mathrm{ppb}$ (geometric $\mathrm{SD}=1.8$ ) to $1.1 \mathrm{ppb}$ (geometric $\mathrm{SD}=2.8, \mathrm{p}=0.01$ ) respectively. The geometric means of Co values remained unchanged. The 10-year survival of Durom THA was $82 \%$, and that of MMC THA $89 \%$ for any revision reason as endpoint.

Interpretation - WB Cr levels decreased over time, and Co levels remained unchanged at long-term follow-up. Despite this we recommend continuing the follow-up of these devices due to relatively low implant survival.
The Durom Metasul large-diameter head (LDH) MoM acetabular device (Zimmer, Warsaw, IN, USA) was introduced in 2003. Overall, there were at least 3,000 implantations in Australia, England, and Finland (1-3). The Durom cup was found to have a high incidence of failure due to lack of osseointegration, and it was recalled in 2008 (4). The Zimmer MMC cup for LDH MoM THA was released in 2009 to address the problems of the Durom cup (5).

After concerns regarding adverse reaction to metal debris (ARMD) emerged, the usage of MMC cups was ceased in 2012 (6). While the revision rate of MoM THA has been relatively high there are still a large number of patients with a MoM device in situ requiring surveillance $(2,7)$. Because ARMD may be asymptomatic, patient-reported outcome measurement (PROM) questionnaires, such as the Oxford Hip Score (OHS), are often not sufficient used alone to detect failing implants (8). Metal artefact reduction sequence magnetic resonance imaging (MARS-MRI) is a reliable tool to detect ARMD, but its utility in screening is limited due to availability, cost, and patient compliance $(9,10)$. Whole blood (WB) metal ions, chromium (Cr), and cobalt (Co), have a fundamental role in the screening of MoM THA patients, although low WB metal ion levels do not exclude ARMD $(11,12)$.

We assessed whether there is any change in the WB metal ion levels in the long term in Durom/MMC THA patients. We also assessed clinical outcomes, risk factors for revision surgery and radiological outcomes of Durom/MMC THA patients. We hypothesized that WB metal ion levels are not increasing during the long-term follow-up of these patients. 


\section{Patients and methods}

We conducted a retrospective cohort study to assess long-term WB Co and Cr level changes and clinical outcomes in Durom and MMC THA patients. Turku University Hospital electronic data pool was used as a data source. We identified all Durom and MMC THAs operated on in our district from March 2005 to January 2011. Unilateral Durom/MMC patients who had a different brand of MoM hip arthroplasty on the contralateral hip were excluded. The patients were followed until revision operation, death, or by the date of October 28, 2019.

We started the systematic screening of MoM hips in 2012, when the ARMD problems became evident. Before that there were only hospital-specific follow-up schedules. The studied implants were not considered to be in a "run in" period when the first ion measurements were performed (13). The screening program is based on the national follow-up protocol recommended by the Finnish Arthroplasty Society. The followup was planned to continue until further notice, based on, e.g., new data available.

According to the screening program all patients with MoM hip arthroplasty should go through clinical examination and/ or a symptom questionnaire, plain radiographs, and WB Co and $\mathrm{Cr}$ ion measurements at least once. If these tests are considered normal, plain radiographs are repeated every 4th year and ion levels every 2 nd year.

Symptomatic patients, and patients with metal ions above 5 $\mathrm{ppb}$, undergo anteroposterior and shoot-through lateral radiographs of the hip every 2 years. In asymptomatic patients with low metal ions these radiographs are taken at a 4-year interval. $\mathrm{Cr}$ and $\mathrm{Co}$ ion measurements and Oxford Hip Score (OHS) questionnaires are checked on a 1- to 2-year interval depending on the symptom state and previous metal ion levels. The OHS questionnaire scale has been presented previously (14). If the patient had a symptomatic hip (poor or moderate postoperative OHS scores), or $\mathrm{Cr}$ and/or Co concentrations above $5 \mathrm{ppb}$, they were scheduled for MARS-MRI imaging of the hip. OHS results of the current study are presented as Supplementary data.

If MARS-MRI is considered normal, these patients undergo clinical examination, symptom questionnaire, and blood metal ion level measurements every year or every 2 nd year depending on the case. Plain radiographs are taken every 2 nd year. Repeated MARS-MRI is recommended if blood metal ion levels increase or there is progression in symptoms.

The details of WB Co and Cr laboratory analyses have been described previously $(\mathbf{1 5}, \mathbf{1 6})$.

We used safe upper limit (SUL) values of $4.6 \mathrm{ppb}$ for $\mathrm{Cr}$ and of $4.0 \mathrm{ppb}$ for Co for unilateral implants. For bilateral implants we used SUL values of $7.4 \mathrm{ppb}$ for $\mathrm{Cr}$ and $5.0 \mathrm{ppb}$ for Co as suggested earlier by Van Der Straeten et al. (17). The number of patients who had Co or Cr ions above the SUL in the repeated measurement were reported.
Table 1. Patient characteristics. Values are $\mathrm{n}(\%)$

\begin{tabular}{lrr} 
& $\begin{array}{c}\text { Operations } \\
\mathrm{n}=249\end{array}$ & \multicolumn{1}{c}{$\begin{array}{c}\text { Patients } \\
\mathrm{n}=227\end{array}$} \\
\hline Age & & \\
$18-59$ & $60(24)$ & $51(23)$ \\
$60-69$ & $87(35)$ & $80(35)$ \\
$\geq 70$ & $102(41)$ & $96(42)$ \\
Sex & & \\
$\quad$ Female & $116(47)$ & $108(48)$ \\
$\quad$ Male & $133(53)$ & $119(52)$ \\
Cup & & \\
$\quad$ Durom & $200(80)$ & $180(79)$ \\
$\quad$ MMC & $49(20)$ & $47(21)$ \\
Bilateral & & \\
$\quad$ No & $205(82)$ & $205(90)$ \\
Yes & $44(18)$ & $22(10)$ \\
Prior operation & & \\
$\quad$ No & $235(94)$ & \\
Yes & $14(6)$ & \\
Anteversion angle & & \\
$>0^{\circ}$ & $236(95)$ & \\
$\leq 0^{\circ}$ & $13(5)$ & \\
Inclination angle & & \\
$0^{\circ}-29^{\circ}$ & $5(2)$ & \\
$30^{\circ}-49^{\circ}$ & $178(71)$ & \\
$\geq 50^{\circ}$ & $66(27)$ & \\
& &
\end{tabular}

All MARS-MRI images available were evaluated by a musculoskeletal radiologist experienced with ARMD-associated pathologies (see Supplementary data). Fluid collections and soft tissue masses were graded by the Hart pseudotumor classification (18). Operative data such as femoral head size and reasons for revision were collected manually from the medical records. The number of metal-related adverse events (pseudotumors, elevated metal ions above the SUL, or revision due to ARMD) were assessed separately for Durom and MMC THAs (see Supplementary data).

\section{Patients}

We identified 227 patients (249 hips) with a Durom or MMC THA. 22 of these patients (44 hips) had a bilateral THA (20 patients with Durom THA and 2 MMC THA). From the 249 hips, there were 126 Durom-CLS, 70 Durom-M/L Taper and $53 \mathrm{MMC}-\mathrm{M} / \mathrm{L}$ Taper THAs. Median age of the patients was 68 years (IQR 14) and 108 (48\%) were women. The median follow-up time for Durom THA was 12 years (IQR 3) and 9 years (IQR 1) for MMC THA (Table 1). 12 patients were revised before metal ion measurements started and 58 patients died during the follow-up.

There were 114 patients with less than 2 repeated metal ion measurements (Durom THA: median 1 [IQR 2], MMC THA: median 2 [IQR 1]). 113 patients had 2 or more metal ion measurements (83 Durom THA patients, 30 MMC THA). The median time elapsing from the index THA to the first metal ion measurement (initial measurement) was 6 years (IQR 4). In staged bilateral patients the median time was calculated from the 2 nd hip replacement operation. The median time from the 


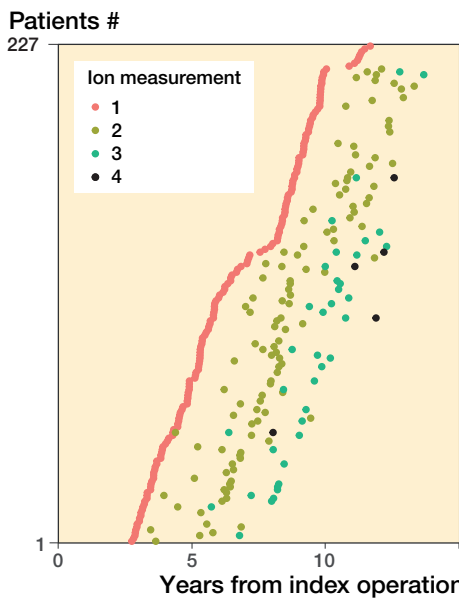

Figure 1. Repeated metal ion measurements by time from operation.
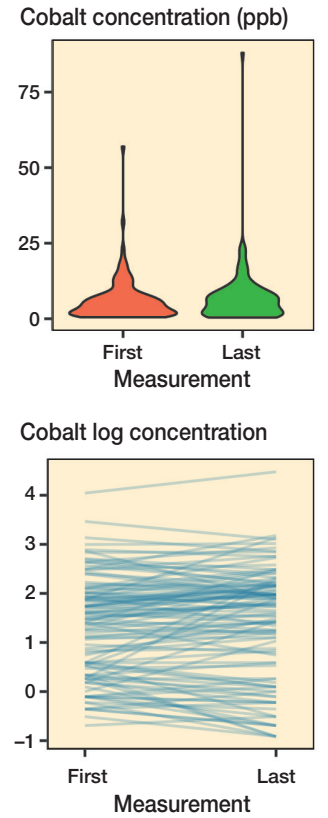

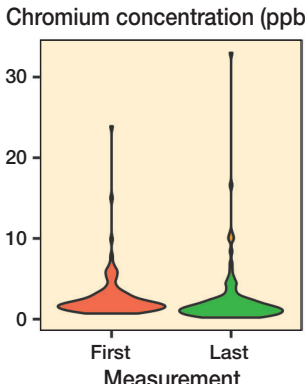

Chromium log concentration

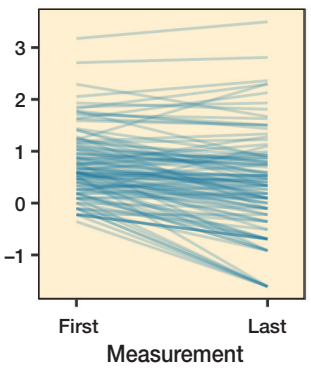

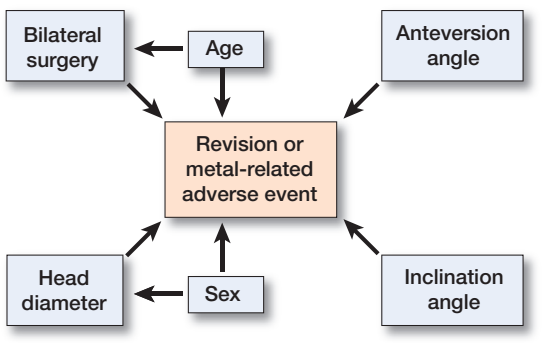

Figure 3. DAG demonstrating the direct causal effects of hip characteristics.

Figure 2. Boxplot figures for initial and control metal ion levels (upper panels). Spaghetti plots for individual cobalt and chromium values at initial and control measurements (lower panels). Values are naturally log-transformed.

introduced, were censored at the time of revision. Furthermore, ion measurements taking place after the revision were excluded from the analysis except for hips that had the same bearing surface even after the revision. For these hips the postrevision ion measurements were also considered in the metal ion level analyses.

Univariable Cox proportional hazards $(\mathrm{PH})$ regression analysis was used to calculate hazard ratios (HR) with 95\% CI for revision for any reason, and separately for any metal-related adverse event (pseudotumor, metal ions above SUL, or revision due to ARMD) (see Supplementary data).

All models were stratified by MoM device. Additionally, we performed multivariable analysis for variables with potential confounding bias by choosing the adjusting variables based on a directed acyclic graph (DAG) analysis.

DAG (Figure 3) was constructed under the following assumptions:

1. Revision surgery or metal-related adverse events are dependent on age, sex, bilateral surgery, inclination angle, anteversion angle, and head diameter.

2. Bilateral surgery is dependent on age because both hips are seldom operated on in the elderly.

3. Head diameter is dependent on sex because head diameter is on average smaller in women.

According to DAG, the estimates for bilateral surgery were adjusted for age and the estimates for head diameter were adjusted for sex. The PH assumption for all Cox models was assessed by a statistical test based on scaled Schoenfeld residuals (19). To achieve the PH function assumption for metalrelated adverse events analysis, cup inclination angle outliers $\left(<30^{\circ}\right.$ or $\left.\geq 50^{\circ}\right)$ were combined to a single outlier group.

$\mathrm{P}$-values below 0.05 were considered statistically significant. Data analysis was performed using the R statistical com- 
Table 2. Metal ion changes with $p$-values for patients with 2 or more metal ion measurements

\begin{tabular}{|c|c|c|c|c|}
\hline \multicolumn{2}{|c|}{ Factor } & Initial & Control & $p$-value \\
\hline \multicolumn{5}{|c|}{ Durom THA $(n=82)$} \\
\hline \multirow[t]{3}{*}{ Co } & Median (IQR) & $5.6(5.5)$ & $6.6(5.4)$ & \\
\hline & Geometric mean (GSD) & $4.6(2.6)$ & $4.9(2.84)$ & 0.2 \\
\hline & Minimum-maximum & $0.5-32$ & $0.4-24$ & \\
\hline \multirow[t]{3}{*}{$\mathrm{Cr}$} & Median (IQR) & $2.0(1.9)$ & $1.5(1.5)$ & \\
\hline & Geometric mean (GSD) & $2.2(1.9)$ & $1.5(2.5)$ & $<0.001$ \\
\hline & Minimum-maximum & $0.7-15$ & $0.2-17$ & \\
\hline \multicolumn{5}{|c|}{ MMC THA $(n=30)$} \\
\hline \multirow[t]{3}{*}{ Co } & Median (IQR) & $1.8(2.2)$ & $2.1(4.8)$ & \\
\hline & Geometric mean (GSD) & $2.2(2.6)$ & $2.3(3.6)$ & 0.6 \\
\hline & Minimum-maximum & $0.6-57$ & $0.4-88$ & \\
\hline \multirow[t]{3}{*}{$\mathrm{Cr}$} & Median (IQR) & $1.6(0.9)$ & $1.1(1.3)$ & \\
\hline & Geometric mean (GSD) & $1.8(1.8)$ & $1.1(2.8)$ & 0.01 \\
\hline & Minimum-maximum & $0.9-24$ & $0.2-33$ & \\
\hline
\end{tabular}

GSD - geometric standard deviation

Values are presented in ppb, which is equal to $\mu \mathrm{g} / \mathrm{L}$.

To convert $\mathrm{ppb}$ of $\mathrm{Cr}$ to $\mathrm{nmol} / \mathrm{L}$ it is necessary to divide by 0.052 .

To convert ppb of Co to $\mathrm{nmol} / \mathrm{L}$ it is necessary to divide by 0.059 .

puting environment version 4.0.3 (R Foundation for Statistical Computing, Vienna, Austria. R packages survival (version 3.2-10) and ggplot2 (version 3.3.3) were used for survival analysis and visualizations, respectively.

\section{Ethics, funding, and potential conflicts of interest}

The study protocol was based on the national recommendation for systematic screening of MoM THA patients given by the Finnish Arthroplasty Society (20). It was a register study, and the patients were not directly contacted. Therefore, approval by the local ethical committee was not needed. Data sharing is not possible. SP has received research funding from Turku University regarding the follow-up of MoM hip arthroplasty patients. MSV reports funding from the Academy of Finland (grant no. 322123). ES, IL, PL , and KTM have nothing to disclose.

\section{Results}

The geometric mean of WB Cr decreased $(\mathrm{p}<0.001)$ in Durom THA patients from $2.2 \mathrm{ppb}$ (GSD 1.9) to $1.5 \mathrm{ppb}$ (GSD 2.5) and in MMC THA patients from $1.8 \mathrm{ppb}$ (GSD 1.8) to $1.1 \mathrm{ppb}$ (GSD 2.8, $\mathrm{p}=0.01)$. The geometric mean of WB Co remained unchanged in Durom THA patients with $4.6 \mathrm{ppb}$ (GSD 2.6) at the initial measurement and $4.9 \mathrm{ppb}(\mathrm{GSD} 2.8, \mathrm{p}=0.2)$ at the control measurement. In MMC THA patients the geometric mean of Co was $2.2 \mathrm{ppb}$ (GSD 2.6) at the initial measurement and $2.3 \mathrm{ppb}(\mathrm{GSD} 3.6, \mathrm{p}=0.6)$ at the control measurement (Table 2 and Figure 2).

Among patients with at least two ion measurements available, 59 patients (53\%, 52 unilateral, 7 bilateral patients) had a Co value above the SUL and 12 patients (11\%, all unilateral)
Table 3. Reasons for revision for both study devices

\begin{tabular}{lcc}
\hline Reasons for revision & Durom THA & MMC THA \\
\hline ARMD & 27 & 3 \\
Periprosthetic fracture & 2 & \\
Loosening of the cup & 6 & \\
Loosening of the femoral component & 2 & \\
Osteolysis & 2 & 1 \\
Infection & 3 & 1 \\
Pain & 2 & 5 \\
Total & 44 & \\
\hline
\end{tabular}

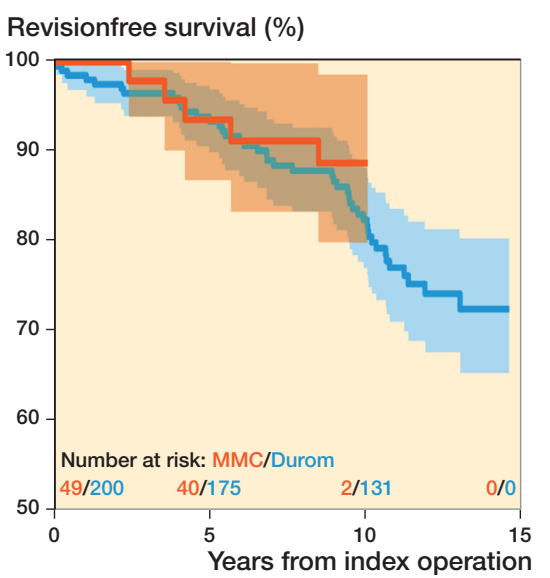

Figure 4. Kaplan-Meier survival curves for both Durom THA and MMC THA with revision for any reason as the endpoint with $95 \% \mathrm{Cl}$.

had $\mathrm{Cr}$ value above the SUL in the first measurement. When the last metal ion measurement was assessed, 65 patients (58\%, 57 unilateral, 8 bilateral patients) had a Co value above the SUL and 10 patients (9\%, all unilateral) had a $\mathrm{Cr}$ value above the SUL. Spaghetti plots for individual WB Co and $\mathrm{Cr}$ changes at initial and control measurement are shown in Figure 2 .

$44(22 \%)$ Durom THAs were revised, and the most common reason was ARMD (27 revisions). 5 hips (9\%) were revised in the MMC THA group and similarly ARMD was most often the reason for revision (3 revisions) (Table 3 ). The 10-year survival of Durom THA was $82 \%$ (CI $0.77-0.88$ ) with any reason for revision as the endpoint. The 10-year survival of MMC THA with any reason for revision as the endpoint was 89\% (CI 0.80-0.99) (Figure 4). Female sex was the only factor that was associated with increased risk of revision $(\mathrm{HR}=2.4$, $\mathrm{p}=0.003$ ) (Table 4).

\section{Discussion}

The main aim of the study was to assess whether there is any change in WB Cr and Co levels in Durom and MMC MoM THA patients over time. WB Cr levels decreased and Co ions 
Table 4. Cox regression analysis data with $95 \% \mathrm{Cl}$ for revision for any reason

\begin{tabular}{|c|c|c|}
\hline Factor & Hazard ratio $(95 \% \mathrm{Cl})$ & p-value \\
\hline \multicolumn{3}{|l|}{ Unadjusted Hazard ratio } \\
\hline \multicolumn{3}{|l|}{ Age (ref. $<60)$} \\
\hline $60-69$ & $1.1 \quad(0.55-2.1)$ & 0.8 \\
\hline$\geq 70$ & $0.81(0.38-1.7)$ & 0.6 \\
\hline Female sex (ref. male sex) & $2.4(1.3-4.4)$ & 0.003 \\
\hline Bilateral surgery (ref. "no") & $0.96(0.46-2.0)$ & 0.9 \\
\hline \multicolumn{3}{|l|}{ Inclination angle (ref. $30^{\circ}-49^{\circ}$ ) } \\
\hline$<30^{\circ}$ & $1.0 \quad(0.14-7.5)$ & 1.0 \\
\hline$\geq 50^{\circ}$ & $0.90(0.47-1.7)$ & 0.8 \\
\hline Anteversion angle $\leq 0^{\circ}$ (ref. $>0^{\circ}$ ) & ') $1.4(0.49-3.9)$ & 0.5 \\
\hline \multicolumn{3}{|c|}{ Head diameter (ref. > $52 \mathrm{~mm}$ ) } \\
\hline $46-52 \mathrm{~mm}$ & $1.7 \quad(0.60-4.8)$ & 0.3 \\
\hline$<46 \mathrm{~mm}$ & $2.9(0.94-8.8)$ & 0.06 \\
\hline \multicolumn{3}{|l|}{ Adjusted Hazard ratio } \\
\hline Bilateral surgery (ref. "no") & $0.95(0.46-2.0)$ & 0.9 \\
\hline $46-52 \mathrm{~mm}$ & $1.3(0.44-3.8)$ & 0.6 \\
\hline$<46 \mathrm{~mm}$ & $1.7 \quad(0.49-5.5)$ & 0.4 \\
\hline
\end{tabular}

In the multivariable analysis bilateral surgery was adjusted for age and head diameter was adjusted for sex. All models were stratified according to MoM THA device.

remained unchanged during the follow-up. The proportion of patients with metal ions above the SUL did not increase during the follow-up. The number of metal-related adverse events was high, but most of these patients did not require revision operation.

While metal ion measurements have a role in the screening of MoM THA patients, there is no international consensus on optimal measurement interval (12). Normal WB metal ion values do not exclude ARMD and elevated metal ion levels do not solely dictate the need for revision surgery. However, elevated metal ion levels are associated with increased wear and further examinations might reveal a failing hip that otherwise might have been missed $(\mathbf{1 1}, \mathbf{1 2})$. The decision to perform a revision operation is also affected, besides ion levels, by the patient's subjective feelings regarding the hip. If the patient is satisfied with the hip, revision surgery is often not needed. Many patients in our study had a surprisingly high OHS score (see Supplementary data). Current guidelines in Finland recommend repeated metal ion measurements every 2 years for all MoM hip devices. Conversely, the FDA does not recommend the routine usage of WB metal ion measurements at all in the screening of MoM patients (21). While the survival of different MoM hip implants varies $(2,3)$, it might also be reasonable to have differing follow-up protocols for each brand from the cost-effective point of view. We have previously stated that if initial WB Cr or Co values are low, repeated metal ion measurement at 2-year intervals does not necessarily provide clinically useful information for patients with unilateral

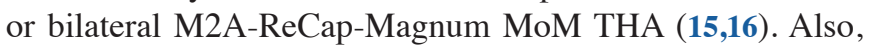
Kiran and colleagues have previously suggested that largehead MoM THA patients might not benefit from annual metal ion measurements if they were asymptomatic (22). We are not aware of any previous reports regarding the repeated metal ion measurements of Durom or MMC MoM THA patients. Our findings advocate that Durom or MMC THA patients do not necessarily benefit from repeated metal ion measurements at 3-year intervals if WB metal ions are initially below the SUL. Our results do not explain why the number of patients with $\mathrm{Cr}$ ion levels above the SUL differs from the number of patients with Co ion levels above the SUL. Further studies are required to explain this discrepancy.

The overall implant survival of Durom and MMC THA in our series was in conformity with previous studies. Seppänen et al. reported in 2018 an overall 10-year survival of $81 \%$ for Durom/MMC THA based on the Finnish Arthroplasty Register (FAR) (23). In an Australian population the 10-year revision rate for the Durom cup was 16\% (2). ARMD was the most common reason for revision in our cohort (13\% of all patients, $59 \%$ of revisions), as expected based on previous registry data (1). Inferior long-term survivorship for Durom/ MMC THA due to ARMD has been reported also in some clinical studies. Ridon et al. reported in 2019 that ARMD was the reason for revision in 29\% of Durom THA patients, with an overall 10-year survival of $67 \%$ (24). Lainiala et al. reported a survivorship of $92 \%$ for Durom THA at 7 years with ARMD as the most common reason for revision $(83 \%$ of all revisions), although they did not separate the Durom and MMC THAs (25). Our data suggests that MMC THA performs slightly better than the Durom THA, although the numbers were small. The 10-year revision rate of Durom-CLS THA is somewhat higher $(25 \%)$ than that of Durom-M/L Taper (12\%) or MMC M/L Taper combination (8.8\%) based on the FAR data. The 10-year revision rate for the M/L Taper stem in conventional THA combinations varies from 5\% to $9 \%$ (3). The better performance of the M/L Taper stem compared with the CLS stem may cause some minor bias to our results.

Inferior survivorship of MoM hip implants in female patients has been reported previously (21). Bilateral surgery, femoral head size, or older age were not associated with an increased risk of revision in our study. Naal et al. have previously reported that femoral head size is not necessarily associated with increased revision risk in Durom HRA patients. Similar to our study, women had a higher revision rate than men (26).

We acknowledge that our study has several limitations. First, our study design was retrospective. Not all the study patients underwent WB metal ion measurements. However, we were also interested in variables and outcomes other than ion levels (such as revision rate). Therefore, we decided to present data of the whole Durom/MMC-group as such, although missing data exists. MARS-MRI was performed only on patients with a symptomatic hip or elevated WB metal ions (above $5 \mathrm{ppb}$ ), and the incidence of pseudotumors in the MRI does not represent the whole cohort. Some of the patients with poor functional outcome may have been revised before participating in metal ion measurements. 
The main aim of our study was to evaluate the change in metal ion levels in repeated measurements. Unfortunately, metal ion level data are not available in the national arthroplasty register. In Finland, most of the revision operations are usually performed in the same hospital district area where the primary implantations were performed. Therefore, we considered that linking our data with the national register would not have altered our results substantially. We think that this bias on our revision rates is minor. We did not have preoperative OHS scores and not all patients filled in a postoperative OHS questionnaire. The radiographic angle measurements were performed in a standard way as described in the Supplementary data, but intra- or inter-class variation was not separately assessed. However, we think this potential bias has only a minor effect on our results.

In conclusion, WB Cr decreased and WB Co remained unchanged in Durom and MMC MoM THA patients. The number of metal-related adverse events was high, but most of them did not require revision.

KTM and MSV designed the protocol and methods. KTM arranged the follow-up of the patients. MSV analyzed the data and did the statistics. SP, ES and KTM collected the data. SP, ES, IL, PL, and KTM wrote the manuscript. All authors contributed to the revision of the manuscript.

Acta thanks Alexander Oxblom and Jörg Schilcher for help with peer review of this study.

1. NJR. National Joint Registry for England and Wales (NJR EnglandWales) 17th Annual Report; 2020. Available from: https://reports.njrcentre.org.uk/Portals/0/PDFdownloads/NJR 17th Annual Report 2020.pdf

2. AOANJRR. Australian Orthopaedic Association National Joint Replacement Registry Annual Report; 2020. Available from: https://aoanjrr. sahmri.com/annual-reports-2020

3. Finnish Arthroplasty Register. https://www.thl.fi/far/.

4. Long W T, Dastane M, Harris M J, Wan Z, Dorr L D. Failure of the Durom Metasul ${ }^{\circledR}$ acetabular component. Clin Orthop Relat Res 2010; 468(2): 400-5. doi: 10.1007/s11999-009-1071-8.

5. Zimmer. Zimmer Holdings Inc. Annual Report; 2009. Available from: https://investor.zimmerbiomet.com/ /media/Files/Z/ZimmerBiomet-IR/ documents/annual-reports/zimmer-2009-ar.pdf.

6. Smith A J, Dieppe P, Vernon K, Porter M, Blom A W, Bsc V, et al. Failure rates of stemmed metal-on-metal hip replacements: analysis of data from the National Joint Registry of England and Wales. Lancet 2012; 379: 1199-204.

7. Lainiala O, Karsikas M, Reito A, Eskelinen A. Whole blood chromium concentration is very rarely elevated independently of whole blood cobalt. Sci Rep 2021; 11(1): 12352. doi: 10.1038/s41598-021-91512-9.

8. Fehring T K, Odum S, Sproul R, Weathersbee J. High frequency of adverse local tissue reactions in asymptomatic patients with metal-onmetal THA. Clin Orthop Relat Res 2014; 472(2): 517-22. doi: 10.1007/ s11999-013-3222-1.

9. Robinson E, Henckel J, Sabah S, Satchithananda K, Skinner J, Hart A. Cross-sectional imaging of metal-on-metal hip arthroplasties: Can we substitute MARS MRI with CT? Acta Orthop 2014; 85(6): 577-84. doi: 10.3109/17453674.2014.964618.

10. Hayter C L, Gold S L, Koff M F, Perino G, Nawabi D H, Miller T T, et al. MRI findings in painful metal-on-metal hip arthroplasty. Am J Roentgenol 2012; 199(4): 884-93. doi: 10.2214/AJR.11.8203.
11. MacNair R D, Wynn-Jones H, Wimhurst J A, Toms A, Cahir J. metal ion levels not sufficient as a screening measure for adverse reactions in metal-on-metal hip arthroplasties. J Arthroplasty 2013; 28(1): 78-83. doi: 10.1016/j.arth.2012.05.029

12. Matharu G S, Mellon S J, Murray D W, Pandit H G. Follow-up of metal-on-metal hip arthroplasty patients is currently not evidence based or cost effective. J Arthroplasty 2015; 30(8): 1317-23. doi: 10.1016/j. arth.2015.03.009

13. DeSouza R M, Parsons N R, Oni T, Dalton P, Costa M, Krikler S. Metal ion levels following resurfacing arthroplasty of the hip: serial results over a ten-year period. J Bone Joint Surg Br 2010; 92-B(12): 1642-7. doi: 10.1302/0301-620X.92B12.24654

14. Mäntymäki H, Junnila $\mathbf{M}$, Lankinen $\mathbf{P}$, Seppänen $\mathbf{M}$, Vahlberg T, Mäkelä K T. Systematic screening of adverse reactions to metal debris after Recap-M2A-Magnum metal-on-metal total hip arthroplasty. Scand J Surg 2017; 106(4): 342-9. Available from: https://doi. org/10.1177/1457496916683093.

15. Pietiläinen S, Mäntymäki $\mathbf{H}$, Vahlberg $T$, Reito $A$, Eskelinen A, Lankinen $\mathbf{P}$, et al. Repeated cobalt and chromium ion measurements in patients with bilateral large-diameter head metal-on-metal ReCap-M2A-Magnum total hip replacement. Acta Orthop 2020; 3674. doi: 10.1080/17453674.2020.1751940.

16. Mäntymäki H, Lankinen $\mathbf{P}$, Vahlberg $\mathbf{T}$, Reito $\mathbf{A}$, Eskelinen A, Mäkelä K. Repeated cobalt and chromium ion measurements in patients with large-diameter head metal-on-metal ReCap-M2AMagnum total hip replacement. Acta Orthop 2019; 90(3): 243-8. doi: 10.1080/17453674.2019.1595469.

17. van der Straeten C, Grammatopoulos G, Gill H S, Calistri A, Campbell P, de Smet K A. The 2012 Otto Aufranc Award: The interpretation of metal ion levels in unilateral and bilateral hip resurfacing. Clin Orthop Relat Res 2013; 471(2): 377-85. doi: 10.1007/s11999-012-2526-x.

18. Hart A J, Satchithananda K, Liddle A D, Sabah S A, McRobbie D, Henckel J, et al. Pseudotumors in association with well-functioning metal-on-metal hip prostheses. J Bone Joint Surg Am 2012; 94(4): 31725. doi: 10.2106/jbjs.j.01508.

19. Grambsch P M, Therneau T M. Proportional hazards tests and diagnostics based on weighted residuals. Biometrika 1994; 81(3): 515-26. Available from: http://www.jstor.org/stable/2337123.

20. Finnish Arthroplasty Society. Recommendation for the use and followup of patients with metal-on-metal (MoM) hip arthroplasty; 2014. Available from: https://www.saply.fi/wp-content/uploads/2020/09/faa_mom_ fu_recommendations.pdf

21. US Food and Drug Administration (FDA). Medical devices: Metalon-metal hip implants, information for orthopaedic surgeons; 2019. Available from: https://www.fda.gov/medical-devices/metal-metal-hipimplants/information-orthopaedic-surgeons.

22. Kiran M, Armstrong C, Shivarathre D, Peter V K. Blood metal ion levels have limited utility in the surveillance of asymptomatic large-head metal-on-metal total hip arthroplasties. J Arthroplasty 2017; 32(12): 3685-8. Available from: https://doi.org/10.1016/j. arth.2017.07.015.

23. Seppänen M, Laaksonen I, Pulkkinen P, Eskelinen A, Puhto A P, Kettunen J, et al. High revision rate for large-head metal-on-metal THA at a mean of 7.1 years: a registry study. Clin Orthop Relat Res 2018; 476(6): 1223-30. doi: 10.1007/s11999.0000000000000159.

24. Ridon P E, Putman S, Migaud H, Berton C, Pasquier G, Girard J. Long-term comparative study of large-diameter metal-on-metal bearings: resurfacing versus total arthroplasty with large-diameter DuromTM bearing. Orthop Traumatol Surg Res 2019; 105(5): 943-8. Available from: https://doi.org/10.1016/j.otsr.2019.04.006.

25. Lainiala O S, Reito A P, Nieminen J J, Eskelinen A P. Declining revision burden of metal-on-metal hip arthroplasties. J Arthroplasty 2019; 34(9): 2058-2064.e1. Available from: https://doi.org/10.1016/j. arth.2019.04.058.

26. Naal F D, Pilz R, Munzinger U, Hersche $\mathbf{O}$, Leunig M. High revision rate at 5 years after hip resurfacing with the Durom implant. Clin Orthop Relat Res 2011; 469(9): 2598-604. doi: 10.1007/s11999-011-1792-3. 


\section{Supplementary data}

\section{Supplementary methods (radiological measurements)}

The cup anteversion and inclination were measured using Carestream Vue PACS software angle measurement tool from standard pelvic AP and lateral shoot-through radiographs. Because the measurement of the anteversion angle may be challenging from lateral radiographs, we categorized the cups into two subgroups, "retroverted" and "not retroverted," for the purpose of regression analysis (numbers and distribution of the data supported this). The measured cup anteversion was compared with the horizontal image plane. Cup inclination was measured from AP pelvis radiographs by drawing a line tangential to the acetabular cup, and another line between the ischial tuberosities. For 18 patients this angle was estimated from AP radiographs of the hip because pelvic radiographs were missing.

The inclination angle subgroups were based on the long-held principle of a "safe zone" for acetabular implants (1). $30^{\circ}-49^{\circ}$ was considered as the optimal inclination (reference). Inclination angles below and above this angle were considered as their own subgroups. There were 97 patients (109 hips) with MARS-MRI imaging of the hip. Postoperative OHS scores were available for 167 patients (183 hips).

\section{Supplementary results}

We identified 97 patients (109 hips) with a postoperative MARS-MRI of the hip. A pseudotumor was found in 66 hips (61\%). The majority (40) were Hart 2A or 2B pseudotumors. Hart 3 pseudotumor was identified in eight hips, and Hart 1 pseudotumor was diagnosed in 18 hips. There were 29 hips with repeated MARS-MRI imaging. Most hips $(\mathrm{n}=29)$ with repeated MRI imaging had undergone 2 MRI imaging sessions, while 2 hips had 3 MRI imaging sessions. 10 patients had normal MRI images in both the initial and repeated MARS-MRI. There were 5 patients with Hart 1 pseudotumor in the initial MRI, but no visible pseudotumor in the repeated MARS-MRI. 3 patients had normal MRI initially but were diagnosed with a pseudotumor in the repeated MRI. 6 pseudotumors increased in size in the repeated MRI but the grade remained the same, and in 1 patient both the grade and size of the pseudotumor increased. 4 patients had a similar pseudotumor on the repeated MRI.

The maximum metal ion values of patients with a MARSMRI were assessed in a subgroup analysis. In cases where MARS-MRI was considered normal, the geometric mean of Co was $7.5 \mathrm{ppb}$ (GSD 2.2). If pseudotumor was diagnosed the geometric mean of Co was 8.9 (GSD 2.5, p = 0.06). Similarly,

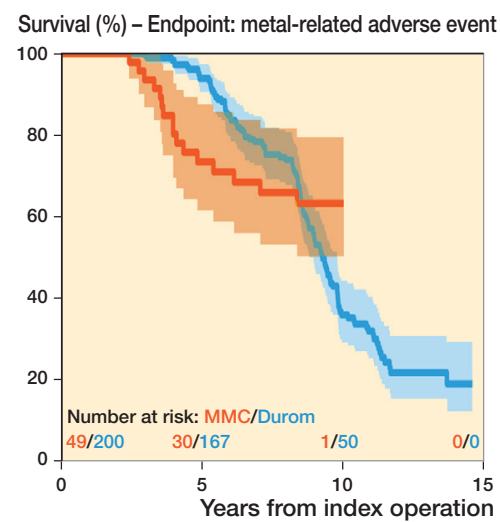

Supplementary Figure. Kaplan-Meier survival analysis for both Durom THA and MMC THA with metal related adverse events as the endpoint with $95 \% \mathrm{Cl}$.

the geometric mean of $\mathrm{Cr}$ in patients with normal MARS-MRI was $2.5 \mathrm{ppb}$ (GSD 2.1) while patients with a diagnosed pseudotumor had a geometric mean of $3.0 \mathrm{ppb}(\mathrm{GSD} 2.2, \mathrm{p}=0.2)$.

The number of metal-related adverse events (pseudotumor, metal ions above SUL, or revision due to ARMD) was 233 in 120 patients (133 hips: Durom 117, MMC 16). A total of 56 patients had one metal-related adverse event, while 30 patients had two metal-related adverse events. Additionally, 34 patients had 3 or more metal-related adverse events. The 10 -year survivorship in terms of metal-related adverse event as the endpoint (with 95\% CI) for Durom THA was 36\% (CI 0.29-0.44), while the 10-year survivorship of MMC THA in terms of metal-related adverse event as the endpoint was $63 \%$ (CI 0.50-0.80) (Supplementary Figure).

167 patients completed the OHS questionnaire postoperatively for 183 hips. One-fourth of the patients reported poor or moderate outcomes (19 [10\%] bad and 20 [11\%] moderate) while the vast majority of the patients had good to excellent outcomes (41 [22\%] good, 103 [56\%] excellent). The mean OHS was 40 (SD 9.8) and the median OHS was 44 (IQR 12). The distribution of OHS scores was similar in both Durom and MMC patients.

Female sex was the only factor that was associated with a higher risk of adverse metal-related events (HR 1.5, $\mathrm{p}=0.03$ ) (Supplementary Table).

\section{Supplementary discussion}

Durom and MMC metal-on-metal (MoM) devices as well as other MoM hip brands were developed to avoid problems with polyethylene wear. Larger head sizes were also associated with 
Supplementary Table. Cox regression analysis data with $95 \% \mathrm{Cl}$ for revision due to adverse reaction to metal debris (ARMD), pseudotumor, Co > SUL or $\mathrm{Cr}>\mathrm{SUL}$ at any point during follow-up

\begin{tabular}{|c|c|c|}
\hline Factor & Hazard ratio $(95 \% \mathrm{Cl})$ & p-value \\
\hline \multicolumn{3}{|l|}{ Unadjusted Hazard ratio } \\
\hline \multicolumn{3}{|l|}{ Age $($ ref. $<60)$} \\
\hline $60-69$ & $0.77(0.50-1.2)$ & 0.2 \\
\hline$\geq 70$ & $0.92(0.60-1.4)$ & 0.7 \\
\hline Female sex (ref. male sex) & $1.5(1.0-2.1)$ & 0.03 \\
\hline \multirow{2}{*}{\multicolumn{3}{|c|}{ Inclination angle (ref. $30^{\circ}-49^{\circ}$ ) }} \\
\hline & & \\
\hline$<30^{\circ}$ & $1.5 \quad(0.16-2.7)$ & 0.6 \\
\hline$\geq 50^{\circ}$ & $1.0(0.66-1.4)$ & 0.9 \\
\hline Anteversion angle $\leq 0^{\circ}$ (ref. $>0^{\circ}$ ) & $1.3(0.67-2.6)$ & 0.4 \\
\hline \multicolumn{3}{|c|}{ Head diameter (ref. > $52 \mathrm{~mm}$ ) } \\
\hline $46-52 \mathrm{~mm}$ & $1.2(0.72-2.0)$ & 0.5 \\
\hline$<46 \mathrm{~mm}$ & $1.6(0.88-2.9)$ & 0.1 \\
\hline \multicolumn{3}{|l|}{ Adjusted Hazard ratio } \\
\hline Bilateral surgery (ref. "no") & $1.1 \quad(0.73-1.8)$ & 0.6 \\
\hline \multicolumn{3}{|l|}{ Head diameter (ref. > $52 \mathrm{~mm}$ ) } \\
\hline $46-52 \mathrm{~mm}$ & $1.1 \quad(0.63-1.8)$ & 0.8 \\
\hline$<46 \mathrm{~mm}$ & $1.3(0.66-2.5)$ & 0.5 \\
\hline
\end{tabular}

In the multivariable analysis bilateral surgery was adjusted for age and head diameter was adjusted for sex. All models were stratified according to MoM THA device.

decreased dislocation revision risk. Both implants were used especially in the young and active population. The Durom cup has a high carbon content and forged chrome-cobalt ( $\mathrm{Cr}-\mathrm{Co})$ alloy structure with optimized clearance. One theory behind the inferior results of the Durom cup was that the rim may cause poor contact with the prepared acetabular bone, which may lead to poor osseointegration and early failure.

We considered that revision rate alone may not adequately represent the rate of functional failure. Therefore, we performed a separate survival analysis in terms of metal-related adverse event as the endpoint (elevated metal ions above the SUL, revision due to ARMD, pseudotumor in MARS-MRI). The 10-year survival of Durom hips was only $36 \%$ while the 10-year survival of MMC hips was $63 \%$.

Even though the amount of metal-related adverse events was high, the majority of the patients reported good to excellent OHS scores postoperatively. All OHS data was collected postoperatively as part of the screening in our study, so we do not have preoperative data. OHS data from all patients at a certain timepoint, e.g., 7 years after operation, is not available. However, the vast majority of the patients reported good to excellent outcomes after Durom/MMC THA implantation, which suggests that patients were mainly satisfied with their total hip.

Since not all patients underwent metal ion measurements or MARS-MRI imaging the true amount of metal-related adverse events might be higher. It is possible that Cox proportional hazards regression analysis could provide slightly different hazard ratios without missing data. Bilateral surgery was associated with an increased risk of metal-related adverse events based on our data, whereas femoral head size or older age were not. The higher rate of aseptic loosening in Durom cups might cause unexpected movement of the cup predisposing implants to edge loading and ARMD $(2,3)$. Cup position was not associated with an increased risk of metal-related adverse events in our study, although the total numbers were small.

In our study, 75 out of 125 (60\%) patients with MARS-MRI had pseudotumor, but direct comparison with other studies is not possible as we performed MARS-MRI only if suggested by the screening protocol. Repeated MARS-MRI changed to normal in many of the initial Hart 1 pseudotumor cases, and the normal finding remained so in most cases. There was no difference in the metal ion levels of patients with a pseudotumor in MARS-MRI and patients with a normal MARS-MRI in the subgroup analysis. However, MARS-MRI was performed only for patients who had symptoms or elevated metal ion levels, which might cause selection bias.

There are 2 previous reports we are aware of concerning incidence of pseudotumors in Durom THA patients. 70 patients of the $102(68 \%)$ in the study of Sutphen et al. (4) and 34 of the 58 (59\%) in the study of Hart et al. (5) had pseudotumor visible in MARS-MRI. The study of Sutphen et al. (4) had mostly Durom patients in their cohort, but the exact number was not reported. Similarly, Hart et al. (5) had also other MoM THA brands than Durom THA included in their study.

Both studied implants (Durom/MMC) have a modular large diameter head that consists of $\mathrm{CoCr}$ alloy. Both stems are monoblock stems that are manufactured from titanium alloy with a similar taper size of $12 / 14$. Modularity of the head provides flexibility for the surgeon, but it creates an additional metal-on-metal interface, where corrosion and wear may occur. Trunnionosis, which refers to corrosion occurring at the head-neck junction in poorly functioning THA implants, can increase the risk of ARMD further. As the head-taper junctions are similar in both implants, we do not believe that trunnionosis causes bias to our study.

1. Lewinnek G, Lewis J, Tarr R, Compere C, Zimmerman J. Dislocations after total hip-replacement arthroplasties. J Bone Jt Surg 1978; 60(2): 217-20.

2. Long W T, Dastane M, Harris M J, Wan Z, Dorr L D. Failure of the Durom Metasul ${ }^{\circledR}$ acetabular component. Clin Orthop Relat Res 2010; 468(2): 400-5. doi: 10.1007/s11999-009-1071.

3. Langton D J, Joyce T J, Mangat N, Lord J, van Orsouw M, Smet $\mathbf{K}$ de, et al. Reducing metal ion release following hip resurfacing arthroplasty. Orthop Clin North Am 2011; 42: 169-80. doi: 10.1016/j. ocl.2011.01.006.

4. Sutphen S A, MacLaughlin L H, Madsen A A, Russell J H, McShane M A. Prevalence of pseudotumor in patients after metal-on-metal hip arthroplasty evaluated with metal ion analysis and MARS-MRI. J Arthroplasty 2016; 31(1): 260-3. doi: 10.1016/j.arth.2015.07.011.

5. Hart A J, Satchithananda K, Liddle A D, Sabah S A, McRobbie D, Henckel J, et al. Pseudotumors in association with well-functioning metal-on-metal hip prostheses. J Bone Jt Surg Am 2012; 94(4): 317-25. doi: $10.2106 /$ jbjs.j.01508. 